\title{
Orthogonal arrays from Hermitian varieties
}

\author{
Angela Aguglia* $\quad$ Luca Giuzzi $^{\dagger}$
}

full screen

close

\begin{abstract}
A simple orthogonal array $O A\left(q^{2 n-1}, q^{2 n-2}, q, 2\right)$ is constructed by using the action of a large subgroup of $\operatorname{PGL}\left(n+1, q^{2}\right)$ on a set of non-degenerate Hermitian varieties in $\mathrm{PG}\left(n, q^{2}\right)$.
\end{abstract}

Keywords: orthogonal array; Hermitian variety; collineation

MSC 2000: 05B25

\section{Introduction}

Let $\mathcal{Q}=\{0,1, \ldots, q-1\}$ be a set of $q$ symbols and consider a $(k \times N)$-matrix $A$ with entries in $\mathcal{Q}$. The matrix $A$ is an orthogonal array with $q$ levels and strength $t$, in short an $O A(N, k, q, t)$, if any $(t \times N)$-subarray of $A$ contains each $t \times 1$-column with entries in $\mathcal{Q}$, exactly $\mu=N / q^{t}$ times. The number $\mu$ is called the index of the array $A$. An orthogonal array is simple when it does not contain any repeated column.

Orthogonal arrays were first considered in the early forties, see Rao [9, 10], and have been intensively studied ever since, see [13]. They have been widely used in statistic, computer science and cryptography.

There are also remarkable links between these arrays and affine designs, see $[12,14]$. In particular, an $O A\left(q \mu_{1}, k, q, 1\right)$ exists if and only if there is a resolvable $1-\left(q \mu_{1}, \mu_{1}, k\right)$ design. Similarly, the existence of an $O A\left(q^{2} \mu_{2}, k, q, 2\right)$, is equivalent to that of an affine $1-\left(q^{2} \mu_{2}, q \mu_{2}, k\right)$ design, see [12].

\footnotetext{
*Research supported by the Italian Ministry MIUR, Strutture geometriche, combinatoria e loro applicazioni.
} 





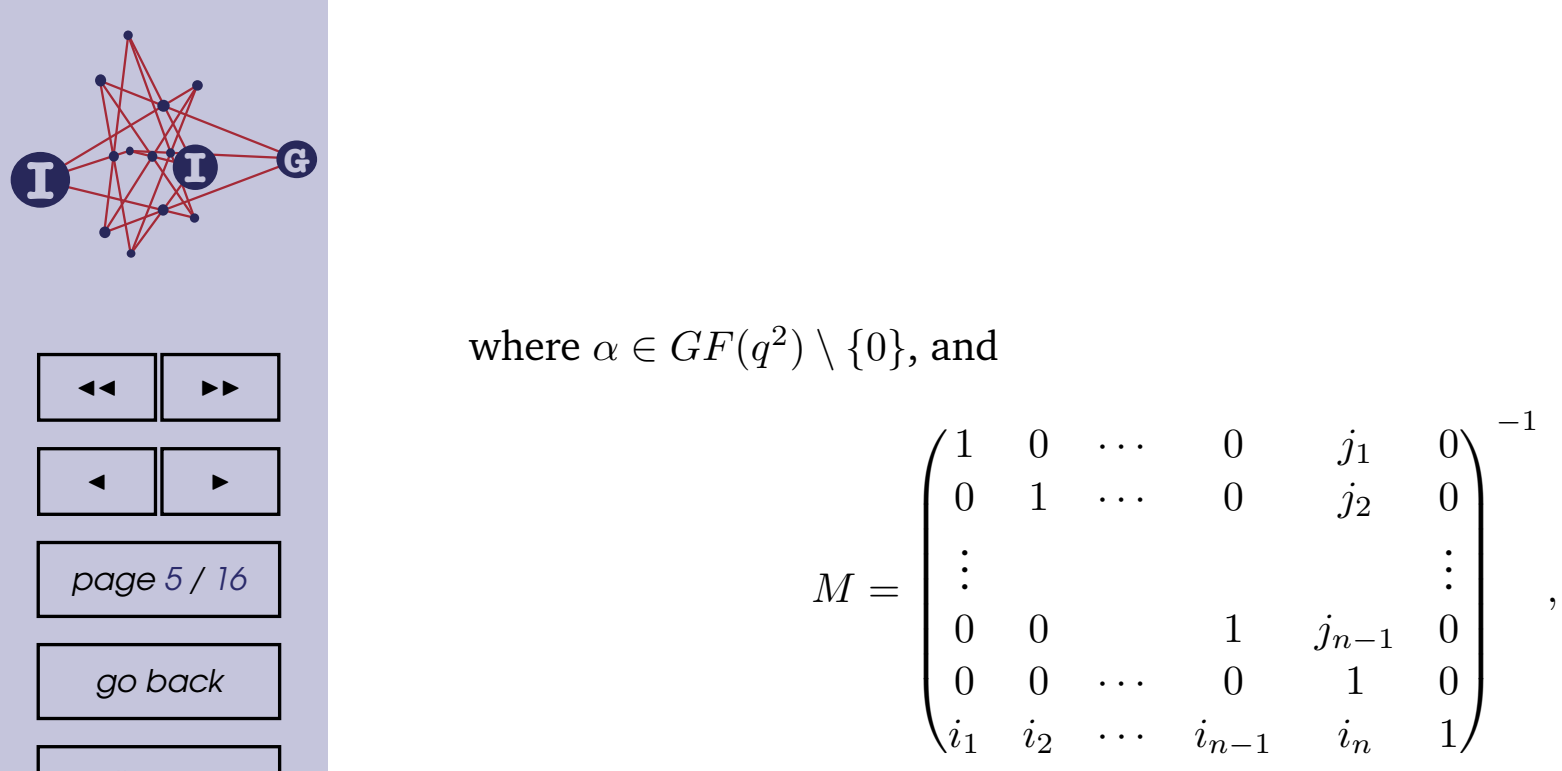

with $i_{s}, j_{m} \in \mathrm{GF}\left(q^{2}\right)$. The group $G$ has order $q^{2(2 n-1)}$. It stabilises the hyperplane $\Sigma_{\infty}$, fixes the point $P_{\infty}(0, \ldots, 0,1,0)$ and acts transitively on $\operatorname{AG}\left(n, q^{2}\right)$.

Let $\mathcal{H}$ be the non-degenerate Hermitian variety associated with the Hermitian form

$$
F=X_{1}^{q+1}+\cdots+X_{n-1}^{q+1}+X_{n}^{q} X_{n+1}+X_{n} X_{n+1}^{q} .
$$

The hyperplane $\Sigma_{\infty}$ is the tangent hyperplane at $P_{\infty}$ of $\mathcal{H}$. The Hermitian form associated with the variety $\mathcal{H}^{g}$, as $g$ varies in $G$, is

$$
\begin{aligned}
F^{g}=X_{1}^{q+1} & +\cdots+X_{n-1}^{q+1}+X_{n}^{q} X_{n+1}+X_{n} X_{n+1}^{q} \\
& +X_{n+1}^{q+1}\left(i_{1}^{q+1}+\cdots+i_{n-1}^{q+1}+i_{n}^{q}+i_{n}\right) \\
& +\operatorname{tr}\left(X_{n+1}^{q}\left(X_{1}\left(i_{1}^{q}+j_{1}\right)+\cdots+X_{n-1}\left(i_{n-1}^{q}+j_{n-1}\right)\right)\right) .
\end{aligned}
$$

The subgroup $\Psi$ of $G$ preserving $\mathcal{H}$ consists of all collineations whose matrices satisfy the condition

$$
\left\{\begin{array}{l}
j_{1}=-i_{1}^{q} \\
\vdots \\
j_{n-1}=-i_{n-1}^{q} \\
i_{1}^{q+1}+\cdots+i_{n-1}^{q+1}+i_{n}^{q}+i_{n}=0
\end{array}\right.
$$

Thus, $\Psi$ contains $q^{(2 n-1)}$ collineations and acts on the affine points of $\mathcal{H}$ as a sharply transitive permutation group. Let $C=\left\{a_{1}=0, \ldots, a_{q}\right\}$ be a transversal of $T_{0}$, viewed as an additive subgroup of $\operatorname{GF}\left(q^{2}\right)$. Furthermore, let $\mathcal{R}$ denote the subset of $G$ whose collineations are induced by

$$
M^{\prime}=\left(\begin{array}{cccccc}
1 & 0 & \cdots & 0 & 0 & 0 \\
0 & 1 & \cdots & 0 & 0 & 0 \\
\vdots & & & & & \vdots \\
0 & 0 & & 1 & 0 & 0 \\
0 & 0 & \cdots & 0 & 1 & 0 \\
i_{1} & i_{2} & \cdots & i_{n-1} & i_{n} & 1
\end{array}\right)^{-1}
$$







by (15) and

$$
\begin{aligned}
& x_{1}^{q+1}+\cdots+x_{n-1}^{q+1}+x_{n}^{q}+x_{n}+x_{1}^{q}\left(a_{1}+b_{1}^{q}\right)+\cdots+x_{n-1}^{q}\left(a_{n-1}+b_{n-1}^{q}\right) \\
+ & x_{1}\left(a_{1}^{q}+b_{1}\right)+\cdots+x_{n-1}\left(a_{n-1}^{q}+b_{n-1}\right)+a_{1}^{q+1}+\cdots+a_{n-1}^{q+1}+a_{n}^{q}+a_{n}=0
\end{aligned}
$$

must have no solution. Arguing as in the proof of (a), we see that this implies that $\left(a_{1}^{q}+b_{1}, \ldots, a_{n-1}^{q}+b_{n-1}\right)=(0, \ldots, 0)$. On the other hand, $P \in \widetilde{B} \cap B^{\prime}$ yields $i_{n}^{q}+i_{n}+a_{1}^{q+1}+\cdots+a_{n-1}^{q+1}+a_{n}^{q}+a_{n}=0$, that is $\omega^{-1} \beta$ is in the stabiliser $\Psi$ of $\mathcal{H}$ in $G$; hence, $B^{\prime}=\widetilde{B}$.

Now, assume $B$ to be a cone-type block. Denote by $\pi$ its basis and let $P^{\prime}=\left(x_{1}^{1}, x_{1}^{2}, \ldots, x_{n-1}^{1}, x_{n-1}^{2}\right)$ be the image $\vartheta\left(x_{1}, \ldots, x_{n-1}, 0\right)$ on the affine space $\mathrm{AG}(2 n-2, q)$ identified, via $\vartheta$, with the affine hyperplane $X_{n}=0$. In $\mathrm{AG}(2 n-$ $2, q)$ there is a unique hyperplane $\pi^{\prime}$ passing trough the point $P^{\prime}$ and disjoint from $\pi$. This hyperplane $\pi^{\prime}$ uniquely determines the block $B^{\prime}$ with property (b).

In order to conclude the proof of the current theorem we shall require a deep characterisation of the high-dimensional affine space, namely that an affine design $\mathcal{S}$ whose parallel classes contain $q>2$ blocks is an affine space if and only if every line consists of exactly $q$ points, see [3, Theorem 12, p. 74].

Recall that the line of a design $\mathcal{D}$ through two given points $L, M$ is defined as the set of all points of $\mathcal{D}$ incident to every block containing both $L$ and $M$. Thus, choose two distinct points in $\mathcal{S}$. As before, we may assume that one of them is $O=N(0, \ldots, 0)$ and let $A=N\left(x_{1}, \ldots, x_{n}\right)$ be the other one.

Suppose first that $A$ lies on the $X_{n}$-axis. In this case, as we have seen before, there are exactly $q^{2 n-3}+\cdots+q+1$ blocks incident to both $O$ and $A$, each of them cone-type. Their intersection consists of $q$ points of $\mathcal{S}$ on the $X_{n}$-axis.

We now examine the case where $A$ is not on the $X_{n}$-axis. As before, we may assume that $A \subseteq \mathcal{H}$, hence (15) holds. Exactly $q^{2 n-3}+\cdots+q+1$ blocks are incident to both $O$ and $A: q^{2 n-2}$ are Hermitian-type, the remaining $q^{2 n-4}+$ $\cdots+q+1$ being cone-type. Hermitian-type blocks passing through $O$ and $A$ are represented by

$$
\begin{aligned}
X_{1}^{q+1}+\cdots+X_{n-1}^{q+1}+X_{n}^{q}+X_{n}+X_{1}^{q}\left(i_{1}+j_{1}^{q}\right)+\cdots+X_{n-1}^{q}\left(i_{n-1}+j_{n-1}^{q}\right) \\
+X_{1}\left(i_{1}^{q}+j_{1}\right)+\cdots+X_{n-1}\left(i_{n-1}^{q}+j_{n-1}\right)=0,
\end{aligned}
$$

with (18) satisfied. Set $x_{s}=x_{s}^{1}+\varepsilon x_{s}^{2}$ for any $s=1, \ldots, n-1$, with $x_{s}^{1}, x_{s}^{2} \in$ $\mathrm{GF}(q)$. The cone-type blocks incident to both $O$ and $A$ are exactly those with basis a hyperplane of $\mathrm{AG}(2 n-2, q)$ containing the line through the points $(0, \ldots, 0)$ and $\left(x_{1}^{1}, x_{1}^{2}, \ldots, x_{n-1}^{1}, x_{n-1}^{2}\right)$. Hence, these blocks share $q$ generators, say $r_{t}$, 


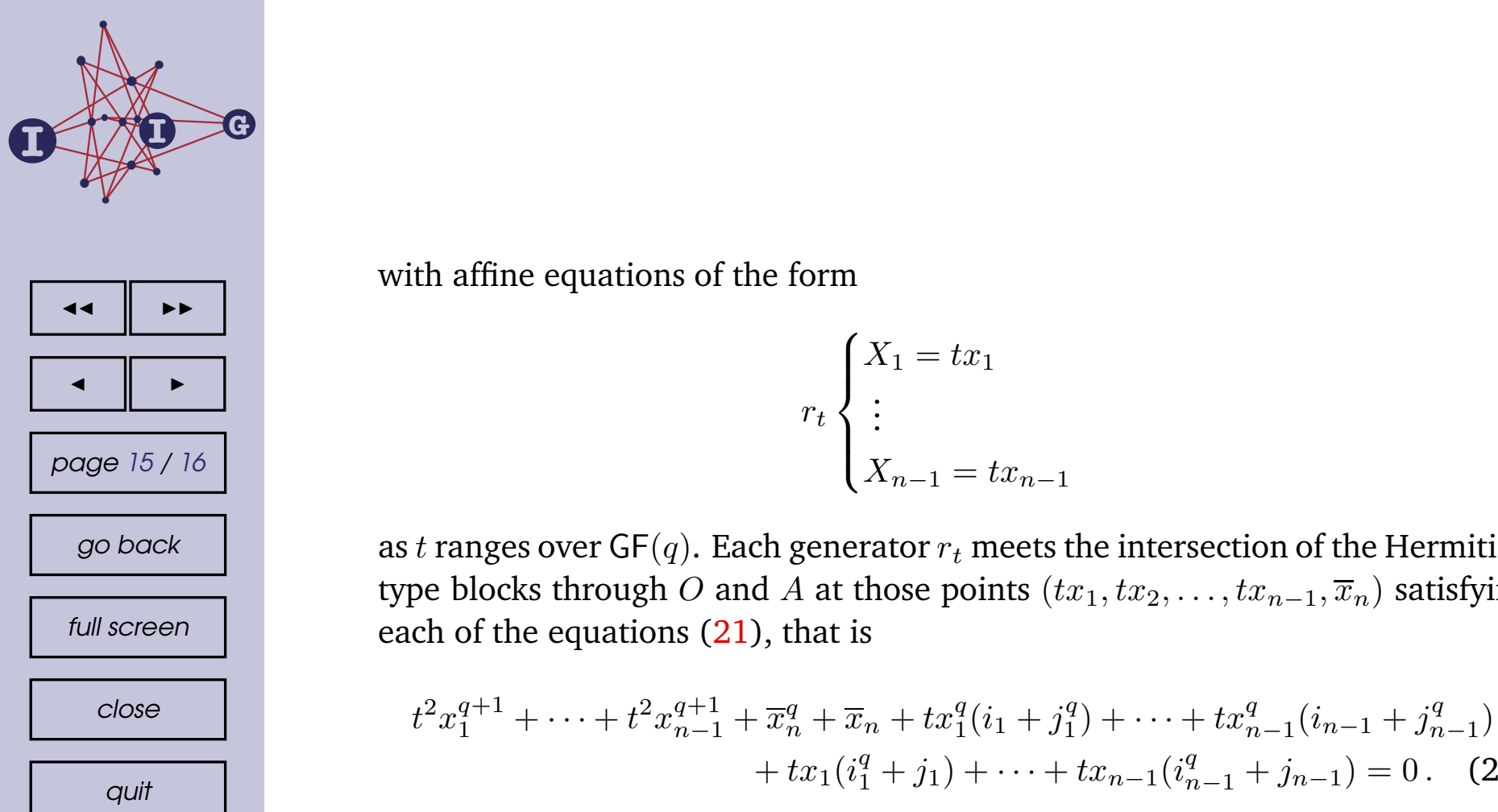

with affine equations of the form

$$
r_{t}\left\{\begin{array}{l}
X_{1}=t x_{1} \\
\vdots \\
X_{n-1}=t x_{n-1}
\end{array}\right.
$$

as $t$ ranges over $\mathrm{GF}(q)$. Each generator $r_{t}$ meets the intersection of the Hermitiantype blocks through $O$ and $A$ at those points $\left(t x_{1}, t x_{2}, \ldots, t x_{n-1}, \bar{x}_{n}\right)$ satisfying each of the equations (21), that is

$$
\begin{aligned}
t^{2} x_{1}^{q+1}+\cdots+t^{2} x_{n-1}^{q+1}+ & \bar{x}_{n}^{q}+\bar{x}_{n}+t x_{1}^{q}\left(i_{1}+j_{1}^{q}\right)+\cdots+t x_{n-1}^{q}\left(i_{n-1}+j_{n-1}^{q}\right) \\
& +t x_{1}\left(i_{1}^{q}+j_{1}\right)+\cdots+t x_{n-1}\left(i_{n-1}^{q}+j_{n-1}\right)=0 .
\end{aligned}
$$

Given (15), (18), equations (22) become

$$
\bar{x}_{n}^{q}+\bar{x}_{n}-t^{2}\left(x_{n}^{q}+x_{n}\right)=0 .
$$

Since $t^{2}\left(x_{n}^{q}+x_{n}\right) \in \mathrm{GF}(q)$, (23) has $q$ solutions, all of the form $\left\{\bar{x}_{n}+r \mid r \in T_{0}\right\}$. The point-set $\left\{\left(t x_{1}, t x_{2}, \ldots, t x_{n-1}, \bar{x}_{n}+r\right) \mid r \in T_{0}\right\}$ coincides with the point $N\left(t x_{1}, t x_{2}, \ldots, t x_{n-1}, \bar{x}_{n}\right) \in \mathcal{P}$. As $t$ varies in $G F(q)$, we get that the intersection of all blocks containing $O$ and $A$ consists, in this case also, of $q$ points of $\mathcal{S}$.

Remark 5.2. The array $\mathcal{A}_{0}$ defined in Theorem 3.3 is closely related to the affine design $\mathcal{S}=(\mathcal{P}, \mathcal{B}, I)$. Precisely, $\mathcal{W}_{0}$ is a set of representatives for $\mathcal{P}$. The rows of $\mathcal{A}_{0}$ are generated by the forms $F^{g}$ for $g$ varying in $\mathcal{R}$, whose associated Hermitian varieties provide a set of representatives for the $q^{2 n-2}$ parallel classes of Hermitian-type blocks in $\mathcal{B}$.

Two orthogonal arrays with the same parameters are said to be equivalent if one can be obtained from the other by permutations of the columns, of the rows, and of the symbols in each column. Since $\mathcal{S}$ is an affine design isomorphic to $\operatorname{AG}(2 n-1, q)$ for $q>2$, it turns out that the array $\mathcal{A}_{0}$ is equivalent to a sub-array of the standard orthogonal array, associated with the classical affine design, as described in $[12,14]$.

\section{References}

[1] A. Aguglia, Designs arising from Buekenhout-Metz unitals, J. Combin. Des. 11 (2003), 79-88.

[2] R. C. Bose, Mathematical theory of the symmetrical factorial design, Sankhya 8, (1947), 107-166. 
